\title{
La verrerie en noir de Trinquetaille à la fin du XVIII e siècle (Arles)
}

\section{Danièle Foy, Henri Amouric}

\section{Résumé}

L'histoire de la verrerie de Trinquetaille à Arles au XVIIle siècle est retracée ici essentiellement à partir des archives et des restes monumentaux de la fabrique. Celle-ci, à l'image de nombreux autres ateliers provençaux de cette époque, doit son développement et sa faillite à une même cause : le charbon. Nous souhaiterions que cette étude, à l'heure où se manifeste un intérêt croissant pour l'archéologie industrielle et pré-industrielle, puisse attirer l'attention sur ces bâtiments en état de ruine, rares vestiges de l'architecture verrière, secteur sans doute non négligeable de l'économie régionale du XVIIle siècle.

\begin{abstract}
This history of the Trinquetaille glassworks in Arles in the 18th Century is here retraced essentially from archives and the monumental remains of the factory. The latter, like many other Provençal workshops of this period, owes its development and collapse to the same cause : coal. We would hope that this study, coming at a time of growing interest in industrial and preindustrial archeology, will draw attention to thèse ruined buildings, rare fragments ofglasswork architecture, a sector no doubt of considérable importance in the régional economy of the 18th Century.
\end{abstract}

\section{Citer ce document / Cite this document :}

Foy Danièle, Amouric Henri. La verrerie en noir de Trinquetaille à la fin du XVIII e siècle (Arles). In: Archéologie du Midi médiéval. Tome 2, 1984. pp. 151-161;

doi : 10.3406/amime.1984.1018

http://www.persee.fr/doc/amime_0758-7708_1984_num_2_1_1018

Document généré le 14/05/2016 


\title{
LA VERRERIE EN NOIR DE TRINQUETAILLE A LA FIN DU XVIII SIECLE
}

(ARLES)

\author{
Henri AMOURIC, Danielle FOY
}

L'histoire de la verrerie de Trinquetaille à Arles au XVIIle siècle est retracée ici essentiellement à partir des archives et des restes monumentaux de la fabrique. Celle-ci, à l'image de nombreux autres ateliers provençaux de cette époque, doit son développement et sa faillite à une même cause : le charbon. Nous souhaiterions que cette étude, à l'heure où se manifeste un intérêt croissant pour l'archéologie industrielle et pré-industrielle, puisse attirer l'attention sur ces bâtiments en état de ruine, rares vestiges de l'architecture verrière, secteur sans doute non négligeable de l'économie régionale du XVIlle siècle.

This history of the Trinquetaille glassworks in Arles in the $18^{\text {th. }}$ Century is here retraced essentially from archives and the monumental remains of the factory. The latter, like many other Provençal workshops of this period, owes its development and collapse to the same cause : coal. We would hope that this study, coming at a time of growing interest in industrial and pre-industrial archeology, will draw attention to these ruined buildings, rare fragments of glasswork architecture, a sector no doubt of considerable importance in the regional economy of the $18^{\text {th. }}$ Century.

\section{INTRODUCTION}

Contrairement à Marseille on ne peut pas dire qu'il y ait de tradition verrière à Arles. Une seule tentative antérieure à l'installation de Trinquetaille est à mettre au crédit de Joseph Dedouard. En 1734, il installe une verrerie artisanale "composé d'un fourneau qui tient deux places» (1) dans laquelle il travaille avec son fils jusqu'en 1740 au moins. Cet atelier est l'exception qui confirme la règle : la ville d'Arles n'a jamais été très propice aux entreprises artisanales et c'est bien souvent grâce à des subventions que l'on est arrivé à en fixer quelques unes. La verrerie Dedouard n'échappe pas à la règle et le Conseil de Ville accorde au maître verrier 100 livres par an «payables de 6 en 6 mois et par avance, laquelle (rente) lui sera continuée tant qu'il restera en cette ville et qu'il y continuera la fabrique» (2). Certaines conditions étaient pourtant favorables à l'implantation de cette industrie : la présence de deux composants principaux du verre: le soude et le sable. En revanche, l'approvisionnement en combustible était difficile dans un terroir peu boisé.

A la fin du XVIIle siècle deux modifications fondamentales interviennent. Les verreries sont de plus en plus souvent alimentées au charbon de terre et le développement du verre noir d'emballage a été fou- droyant. La plupart des liquides sont à cette époque conservés et transportés dans des conteneurs de verre épais et résistant. La demande intérieur et extérieure a cru de façon considérable. La fabrique d'Arles s'inscrit dans cette logique ; elle utilise les ressources naturelles locales (sable grossier et soude) comme les possibilités offertes par la présence des voies d'eaux : arrivée du combustible par le Rhône, diffusion de la production par le fleuve et les canaux (Arles à Sète et canal du midi) vers les marchés réexpéditeurs régionaux et internationaux (3).

Les hasards de la conservation font qu'il subsiste encore aujourd'hui une partie des archives de la verrerie de Trinquetaille (4). De plus certains bâtiments de la fabrique ont survécu. C'est essentiellement à partir de ces données que nous avons conduit notre étude.

\section{HISTORIQUE}

L'histoire de la verrerie de Trinquetaille commence le 3 février 1781 par la signature du bail de l'enclos Datty (5) qui engage Jacques Grigniard de la Haye (6), François Brun et Pierre Boulouvard l'aîné dans la perspective de l'installation d'une verrerie. Il leur fallut au préalable obtenir l'autorisation du Conseil d'Arles qui leur fut accordée le 10 mars 1782 (7). Des tractations qui précédèrent cette demande nous ignorons tout.

(1) A.D. 13. C 2301 fos $18-19$

(2) A.C. Arles, BB 47, 1734.

(3) Les entrepreneurs avaient peut-être en tête le projet de la route provence-Languedoc. A.N. F 12/1490 requête du sieur Jean Datty 5 avril 1785.

(4) Bibl. Mun. Arles, ms. 958.

(5) A.D. $13401 \mathrm{E} 422 \mathrm{f}^{\circ} 11$.

(6) J. Grigniard de la Haye est originaire de Champeneuville en Normandie, du Comté d'Eu, célèbre pour ses verreries. A.D. 13, C3419, pièce pour 1785.

(7) A.C. Arles, BB 55 , fos $67-68$. 


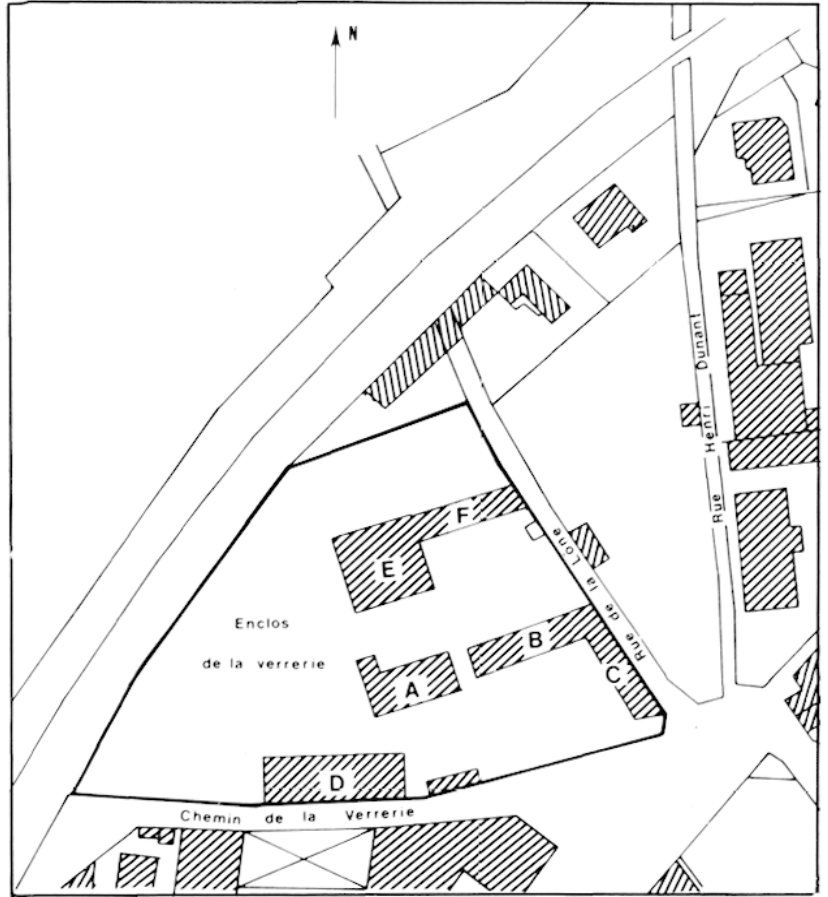

Fig. 1 - Plan de situation des bâtiments et de l'enclos d'après le cadastre de 1963. E : halle; A : bureau (?) ; B : logement d'ouvriers : $C, F$ et $D$ : bâtiments postérieurs.

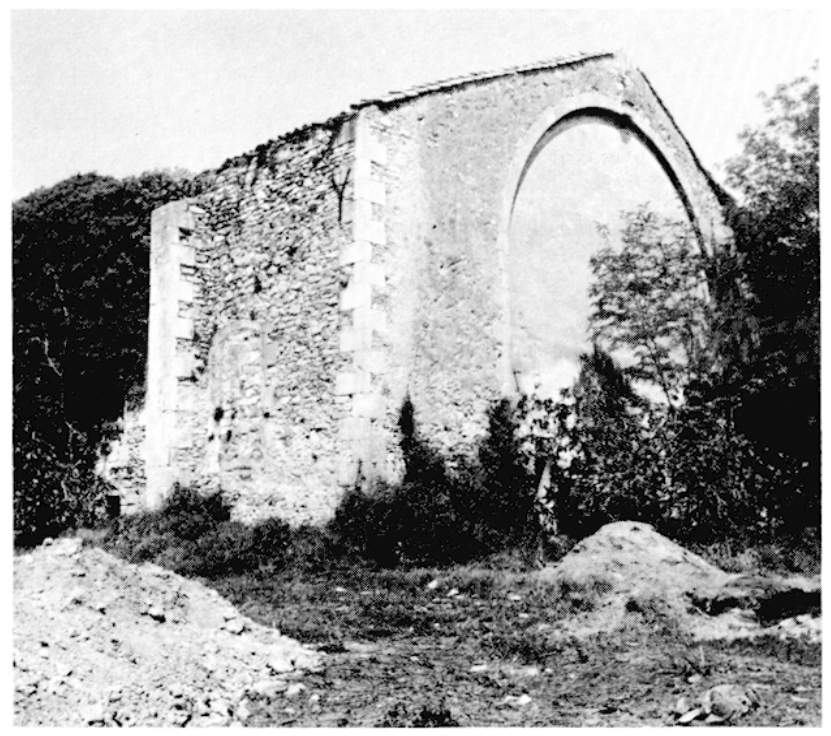

Fig. 2 - Vue d'ensemble de la halle prise du Sud-Ouest (document inventaire ; cliché Roucaute-Heller).
Dès le 14 mars suivant un acte de société en bonne et due forme est dressé entre les trois compères sous la raison sociale Grigniard et $\mathrm{Cie}$, en toute logique d'ailleurs puisqu'il était le seul homme de métier (8). Le 20 mars 1782 un nouveau bail de 9 ans leur est accordé par le sieur Datty (9). D'ores et déjà une possibilité d'extension est prévue. L'arrangement à peine paraphé, la discorde s'installe parmi eux, le sieur Boulouvard «après avoir appris de ses associés tous les renseignements concernant la dite verrerie avoit sollicité le dit privilège en son seul nom dans la vue de former en son particulier l'établissement d'une autre verrerie en profitant de la correspondance de la société établie sous son nom et occasionner par là la ruine de ses associés» (10). La manœuvre réussit parfaitement et le 29 octobre 1782, le Conseil du Roi lui octroie l'autorisation sollicitée (11). Quelle qu'ait été l'issue de cette intrigue, il ne semble pas qu'elle ait eu de conséquence importante puisque l'association perdura jusqu'en 1791. Le sieur Grigniard remplit ses fonctions sans discontinuer comme en témoignent les registres de capitation des années $1783,84,87$ et 88 (12). En dépit d'une information très lacunaire (pour les années 1780) qui ne nous permet pas d'apprécier la prospérité de l'affaire, le fonctionnement de la fabrique devait être satisfaisant puisqu'en 1782 et 1783 les associés font lacquisition de trois parcelles jouxtant l'enclos en vue d'agrandissement (13). Peut-être estce pour la même raison que le sieur Datty essaye en 1785 de faire casser le bail qui le liait pour 9 ans (14). En vain apparemment. Quelques mois après cette tentative, il cède à la société l'enclos et les bâtiments de la verrerie y compris ceux construits aux frais des associés (15).

En 1791, à l'échéance du contrat de société, la fabrique change de mains, malgré l'opposition des Sieurs Brun et Grigniard. Cession en est faite à Trophime Tinellis, Paul Agricol Bonnard, Siffrein Boulouvard et Joseph Yvaren notaire qui se forment aussitôt en société (16). La vente n'est pas effectuée au comptant et certaines créances sont reportées des premiers acheteurs sur les seconds (17). Dans le souci d'apurer les comptes, les nouveaux sociétaires procèdent au rachat de la pension féodale qui portait sur le sol de l'enclos (18). L'activité de la manufacture se poursuit sans à coup discernable jusqu'en 1793.

En 1793 vraisemblablement, Joseph Yvaren devient le seul maitre des lieux (19). Sans que l'on sache pourquoi, l'activité est ensuite interrompue jusqu'en l'an V. L'entreprise est relancée pendant une

(8) A.N. F $12 / 1487$.

(9) A.D. 13401 E $422 f^{\circ} 142$.

(10) A.N. F 12/1487, mémoire des sieurs Brun et Grigniard, 1782

(11) Ibid. Arrêt du conseil.

(12) A.C. Arles. CC 119. fos $284 v^{\circ}$ et 599. Capitations de 1783 et 1784 ; ibid. CC 121 fos 199 et 456 . Capitations de 1787 et 1788.

(13) A.D. 13, $404 \mathrm{E} 1190 \mathrm{f}^{\circ} 456$ v. Origine de propriété achat aux sœurs Le Blanc 16 auril 1782, achat au sieur Michel 27 novembre 1782 ; achat à Alexandre Ganteaume 16 septembre 1783 .

(14) A.N. F 12/1490.

(15) A.D. 13401 E $407 f^{\circ} 169$. Vente intervenue le 10 octobre 1785.

(16) A.D. 13404 E $1190 f^{\circ} 456 v^{\circ} 24$ septembre 1791 et A.D. 13 C 3419

(17) Bibl. Mun. Arles, ms. $958 f^{\circ} 13 v^{\circ}, n^{\circ} 8$ et $f^{\circ} 14 v^{\circ}$

(18) lbid. $f^{\circ} 13 v^{\circ}, n^{\circ} 9$.

(19) lbid. $f^{\circ} 21$ 
brève période qui s'achève avec la journée du 18 fructidor an V. Spolié de ses biens, Yvaren ne remet la manufacture en route qu'en l'an VIII lors de sa restitution ; il semble alors avoir cédé une part de la fabrique à son ancien fermier Gabriel Blanchet (20). En l'an XI l'atelier est pourtant abandonné face à une conjoncture très défavorable : imposition de 20 centimes par 5 myriagrammes de charbon de terre, taxe de 30 francs par journée de travail, blocus continental qui le prive du débouché essentiel, dit-il, des Antilles françaises et accessoirement de celui de l'ltalie. Caressant toujours l'espoir de la remettre en service, Yvaren attent 1809 pour liquider le matériel de la verrerie à "Tubœuf Frères propriétaires des mines de charbon et de la verrerie d'Alais» (21).

A sa mort, en 1825 l'enclos et le bâtiment sont toujours dans le patrimoine des Yvaren (22). Son fils Henri l'obtient en partage de la succession paternelle en 1829 (23). Un rapport d'expert nous apprend que certains bâtiments sont déjà détruits et d'autres en mauvais état (24). En 1834, Henri Yvaren demande et obtient l'autorisation de rallumer ses fours. Cette ultime tentative ne doit guère avoir dépassé le stade des intentions (25). La verrerie en noir de Trinquetaille est alors définitivement abandonnée.

\section{LA FABRIQUE}

\section{- Les bâtiments}

Il n'existe pas à notre connaissance de plan de la verrerie ni de description détaillée. Il faut donc se contenter de données éparses et du peu qui subsiste pour évoquer l'ensemble complexe qu'elle formait. Les montants des transactions qui sont élevés, indiquent clairement l'importance de la manufacture. En 1785, la vente du seul enclos Datty se fait pour un prix de 11000 livres (26). En 1791, la totalité de la verrerie est cédée pour 22050 livres (27). En 1828 encore, alors que beaucoup de bâtiments d'exploitation sont détruits, le rapport d'expertise estime à 10000 francs ce qui reste debout. Les constructions importantes n'ont pas été réalisées en une seule fois.

De la première verrerie qui réutilisait, au moins partiellement, des constructions réalisées par le sieur Datty dans son enclos, nous ne savons pas grand chose sinon qu'une première halle a été édifiée dans le courant 1781 ou 1782 à l'intérieur même de l'enclos, Le bail du 3 février 1781 , confirmé par celui du 20 mars 1782 le prévoyait explicitement : "comme les dits preneurs se proposent d'établir une fabrique de verrerie dans ledit enclos et battiment et que le dit bas-

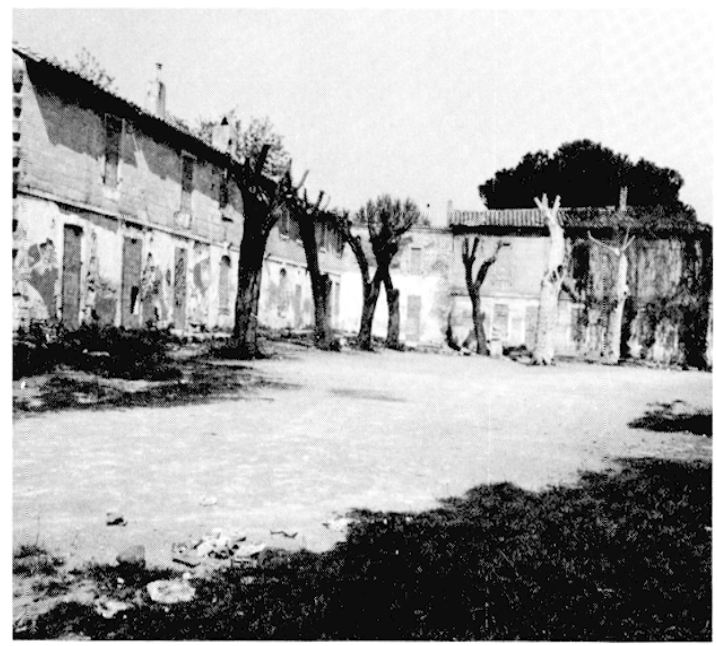

Fig. 3 - Vue d'ensemble des corps des bâtiments $B$ et $C$ prise de l'Ouest (document inventaire ; cliché Roucaute-Heller).

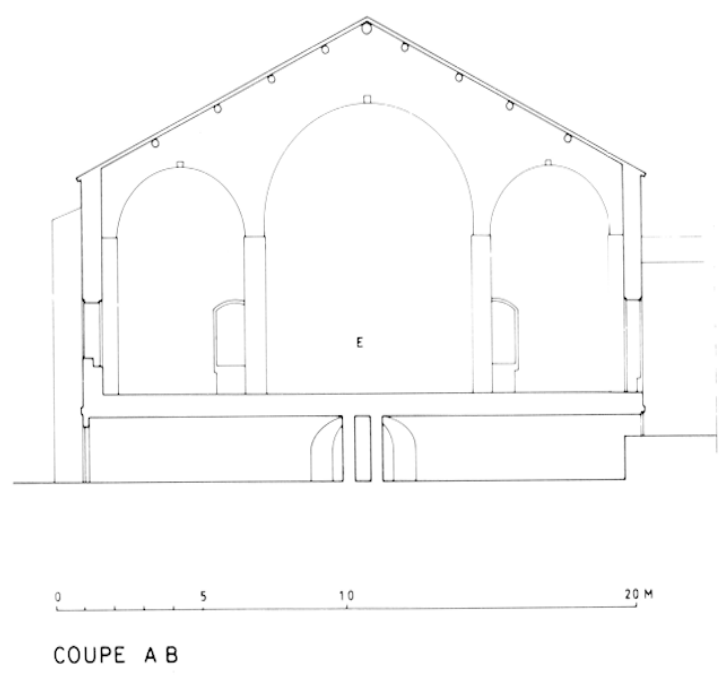

Fig. 4 - Elévation de la halle (document inventaire; relevés PEGAND-FRAY).

timent n'est pas suffisant pour cet objet, il leur sera permis d'en édifier un autre dans tel endroit dudit enclos qui leur sera le plus convenable pourvu qu'il ne soit pas adossé a la façade du battiment actuel du côté du midi» (28).

Une seconde halle "contigüe à la première" est édifiée par la suite sur des terrains acquis en 1782 . 1783. Elle est mentionnée dans les confronts de la cession Datty en 1785 "Scavoir est l'enclos dans partie

(20) Ibid. $f^{\circ} 9$ et $f^{\circ} 53$.

(21) Ibid. fo 67 à fo 82 .

(22) Bibl. mun. Arles, ms 950 p. 13 ; 2 février 1825 Testament de maitre Joseph Yvaren qui lègue entre autre «l'enclos terrein et batiment de mon ancienne verrerie en noir de Trinquetaille.

(23) Ibid. ms 949 p. 114 et 115 , traité entre les hoirs Yvaren pour la vente de la verrerie. 28 juillet 1828 et p. 119 attribution de l'enclos à Henri Yvaren par jugement du 13 février 1829.

(24) A.D. 13306 U 12, Tribunal de Tarascon pièce du 13 octobre 1828.

(25) A.D. 1314 M 12/355 :

(26) A.D. 13401 E 407 f० 169.

(27) A.D. 13404 E $1190 f^{\circ} 456 v^{\circ}$

(28) A.D. $13401 \mathrm{E} 422$ f $^{\circ} 111$ et 142. 


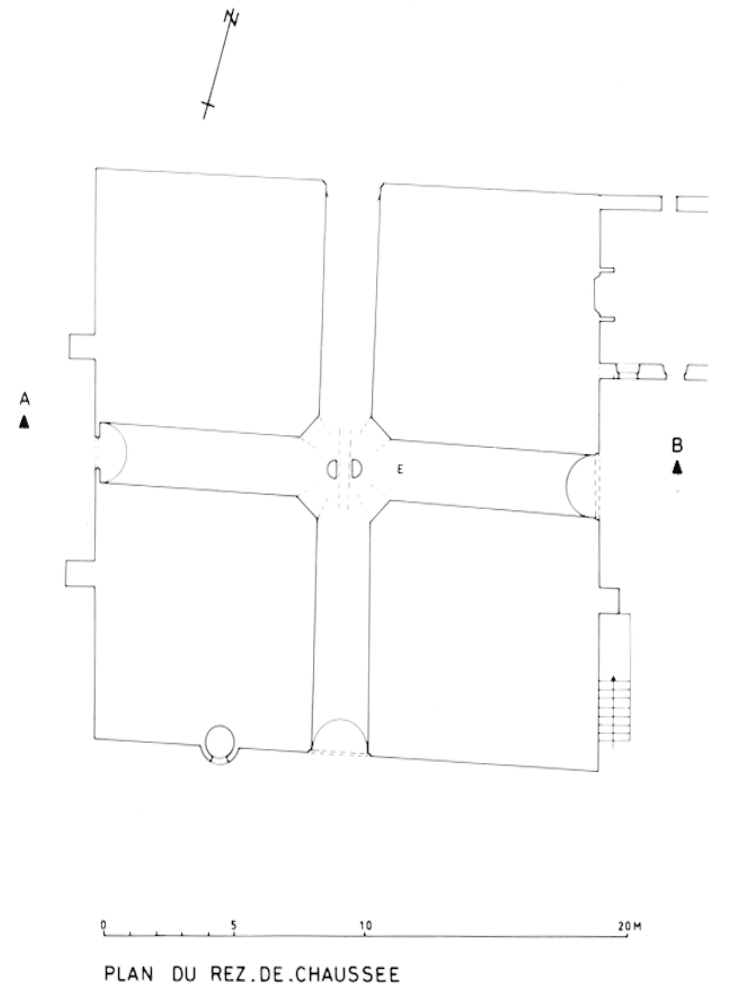

Fig. 5 - Plan du demi sous-sol (document inventaire) ; relevés PEGAND-FRAY)

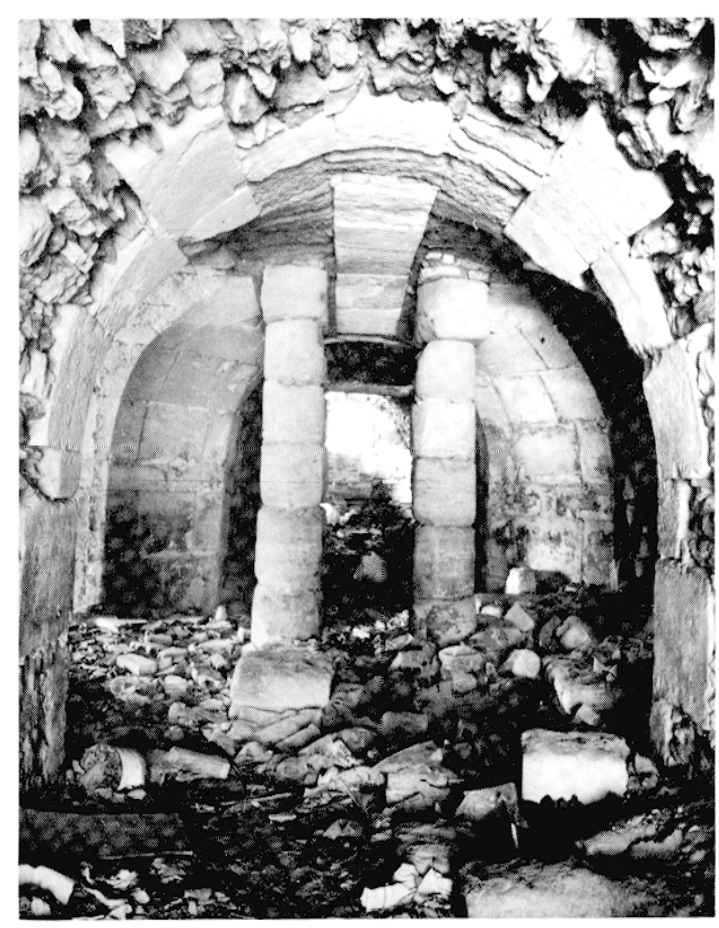

Fig. 6 - Intersection des deux couloirs de tirage (document inventaire ; cliché ROUCAUTE-HELLER). duquel est construit un des fourneaux de cette verrerie et autre bâtiment... du nord bâtiment et second fourneau de cette verrerie, construit sur un emplacement enclos de muraille...» (29). La halle a demi-ruinée qui subsiste encore est certainement la construction la plus récente (fig. 2). Le claveau de l'arc central de la première travée porte gravée la date de 1785 . La manufacture est encore aujourd'hui inscrite dans un enclos irrégulier bordé au Sud par le chemin de la verrerie, à l'Est par la rue de la Lône et sur les autres côtés par la voie ferrée qui a empiété sur le domaine (fig. 1 et 2). De l'ensemble originel subsite uniquement la halle, un corps de logement d'ouvriers et un bâtiment qui servait peut-être de bureaux. Les portes, fenêtres, arcs, chambranles (fig. 3), chaînages d'angles, cloisons et élévations de la façade sont appareillés en calcaire jaunâtre clair (probablement extrait des carrières de Beaucaire) (30). La halle, de plan grossièrement carré, comporte deux niveaux (fig. 4). Un demi soussol composé de deux couloirs voûtés en berceau, se coupant en croix au milieu de l'édifice et ouvrant à l'extérieur au centre de chaque façade, supporte l'élévation (fig. 5). Les couloirs sont construits en blocage mais les arêtes de pénétration des voûtes sont appareillées. Une partie de la voûte centrale a été remaniée. Deux piliers semi-cylindriques, reliés par une barre de fer, sont construits au milieu de l'intersection. $\mathrm{Ce}$ dispositif est caractéristique des verreries à charbon. L'Encyclopédie mentionne ces caves qui font office de tirage. Les piliers servaient à soutenir lés sièges du four sur lesquels étaient posés les creusets, Entre les piliers était située une grille qui formait le fond du foyer (fig. 6).

L'étage sous charpente est divisé en trois nefs orientées est-ouest, par des arcs en plein cintre (fig. 7 et 8). Il ne reste rien des aménagements intérieurs. On sait seulement que le four de fonte occupait le centre de cet espace. Il est possible d'imaginer qu'à Arles, comme ailleurs, les fours de recuit occupaient les angles de la bâtisse. La façade sud était presque entièrement ouverte par un arc en plein cintre aujourd'hui muré. Les façadé est et ouest sont épaulées de contreforts qui n'ont cependant pas empêché un certain devers de l'ensemble (31).

La manufacture comprenait aussi d'autres édifices: des logements d'ouvriers, un bureau pour l'administration (32) comme nous l'avons déjà dit, des annexes d'exploitation, magasins divers pour les matières premières et les produits finis, une cave, une forge pour les ouvrages métalliques (33), une pillerie pour les écailles de creusets et la terre brûlée entrant dans la confection des creusets, un four à chaux et peut-être un atelier pour le potier, le tout délimitant une cour. Il n'est pas certain qu'ils aient été suffisants puisqu'il fut question de se "procurer un magasin à Arles pour les cendres» (34).

(29) A.D. 13401 E $407 f^{\circ} 169$.

(30) Nous avons plusieurs mentions d'achats de pierres de Beaucaire pour la verrerie. En l'an $V$ par exemple $\times 122$ pierres de Beaucaire de 4 pans longueur, 2 pans largeur, un pan hauteur» sont acquises pour la construction d'un nouveau hangar, Bibl. mun. Arles, ms $958, f^{\circ} 27$.

(31) La verrerie de Trinquetaille a fait l'objet d'un dossier de la Commission Régionale d'Inventaire, Provence-Alpes-Côte d'Azur.

(32) Bureau mentionné dans A.D. $13404 \mathrm{E} 1190 \mathrm{f}^{\circ} 456 \mathrm{v}^{\circ}$.

(33) Bibl. mun. Arles ms $458, f^{\circ} 78$.

(34) Ibid. $f^{\circ} 61$. 


\section{- Les fours}

Dans chacune des halles se trouvait un four de fusion et un nombre inconnu de fours de recuits qui ne devait cependant pas être inférieur à 4 si l'on en croit un «aperçu des dépenses et produits d'un four de verrerie à l'allemande pour verre blanc» correspondant à un projet des propriétaires (35). Ce chiffre n'est cependant que pure hypothèse, l'état des dépenses de 1792-93 mentionne seulement 4 ouvriers soufleurs et un de relai, donnée qui plaide plutôt pour un petit nombre de fours de recuit. Il est également possible qu'un seul des fours ait continué à fonctionner à cette époque (36).

La confection des fours requiert des briques, de formes, de tailles et d'appellations diverses : briques de couronne pesant 40 livres l'une, briques carrées de 80 l, lits de camps de 150 l. Ces pièces très lourdes sont toutes fabriquées et moulées sur place (37) à partir de terres réfractaires de bonne qualité amenées de Bédoin (Vaucluse) ou de Salevas (Ardèche ou Isère) (38). Les fours sont des instruments de travail coûteux : une simple réfection à neuf est estimée à 2000 livres pour une durée de vie approximative de 8 ans (39). La construction d'un four neuf est évaluée 3000 livres et plus (jusqu'à 3718 livres) pour une longévité de 6 ans 1/3 non comprises d'indispensables répara. tions annuelles dont la valeur est diversement appréciée selon les documents : 601 par mois environ en 1792-93, 40 I par an seulement, (mais pour la réfection des sièges uniquement), dans un autre cas.

\section{- Les creusets et le travail des terres}

Au chapitre des dépenses importantes figurent les "pots», dont la fabrique faisait une grande consommation. L'état de 1791 (40) prévoit le remplacement de 10 pots par mois de campagne, celui des dépenses courantes de 1792-93 (41) comptabilise la «matière de 15 creusets" pour une même durée d'exploitation. Ces creusets sont apparemment assez importants : 2 quintaux (environ $80 \mathrm{~kg}$ ) pièce dans le projet de la verrerie à l'allemande (42) et 380 livres (190 kg environ) pour la campagne 1792-93 ; ils sont aussi assez coûteux : de 20 à 25 livres l'un (43). D'aussi grosses pièces (44), nécessitèrent d'importantes quantités de terres d'origines diverses; on utilise de préférence les mêmes que celles qui entrent dans la composition des

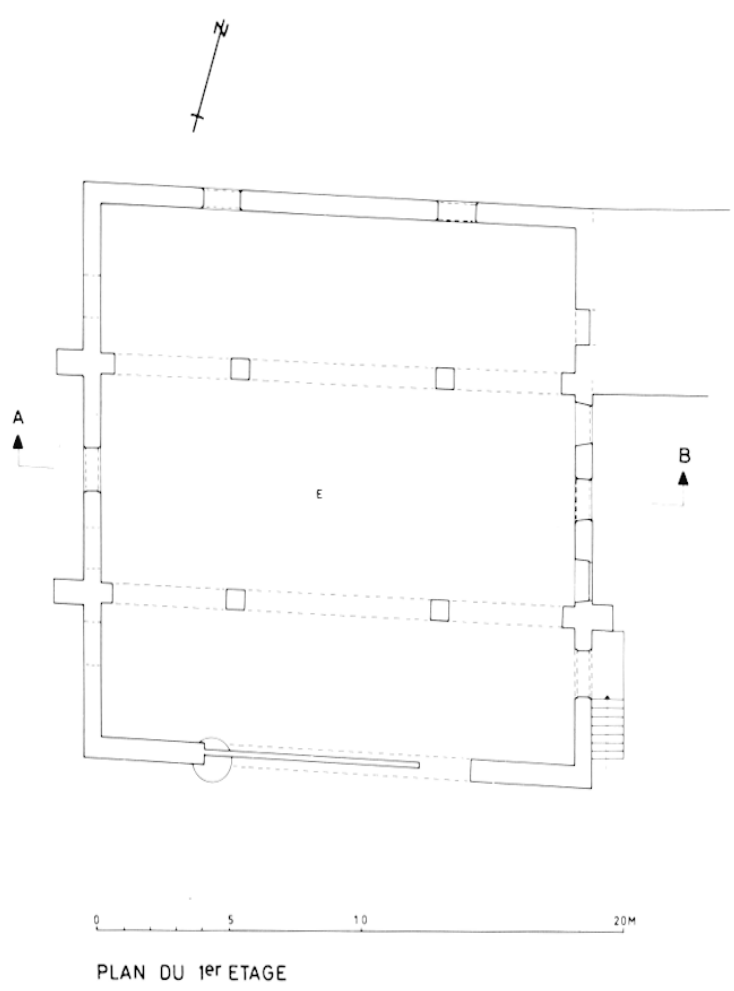

Fig. 7 - Plan de l'étage (document inventaire; relevés PEGAND. FRAY).

fours : terres de Bédoin et de Salavas dont les qualités réfractaires reconnues assurent la célébrité (45) et expliquent le prix : 3 livres le quintal à l'état brut et de 5 à 8 livres après traitement. Mais aussi "terre grasse» peut être extraite d'une propriété acquise en 1791 par les exploitants de la verrerie, en Crau au quartier de Fourchion (46).

Pour confectionner un pot, on mélange une argile crue et une ou plusieurs argiles cuites, afin de diminuer les risques de fissuration. A Trinquetaille, les argiles cuites sont obtenues à partir de terres de Salavas et de Bédoin que l'on fait brûler au préalable, puis piler et d'écailles de vieux creusets dépouillées de leur vernis, réduites en poudre. La composition du mélange est en général inconnue ; en 1792-93, toutefois les proportions indiquées sont les suivantes : «30 livres d'écailles de vieux creusets, $200 \mathrm{~L}$ de terre de

(35) lbid., $f^{\circ} 17$.

(36) Lors de la cession de 1791 il est dit que les bâtiments sont en mauvais état, peut être un des fours est-il abandonné à cette époque.

(37) Bibl. mun. Arles ms $958 f^{\circ} 78$ « 7 moules pour les briques de différentes formes». L'Encyclopédie donne une idée exacte de ces briques et de ces moules.

(38) La localisation de Salavas pose un problème. L'enquête des préfets de 1809 au Musée de Sèvres mentionne pour le Vaucluse «il n'existe plus de verrerie dans ce département et il n'est plus possible d'y trouver de la terre de Salavas que l'on y employait. On faisait venir celle terre de la commune de Vorep département de l'Isère». Par contre pour le préfet du Gard ales sables et terres dont on se sert pour les creu. sets ne se tirent pas des environs de Pont-Saint-Esprit. Ils viennent de Salavas (département de l'Ardèche)".

(39) Bibl. mun. Arles, ms 958 fos 36-37.

(40) Ibid. $f^{\circ} 46$

(41) lbid. fos $36-37$.

(42). Ibid. $f^{\circ} 17$. Pour une campagne, il est prévu une centaine de pots.

(43) Les creusets de la campagne 1792-1793 sont composés chacun de «30 livres d'écailles de vieux creusets : 12 sous 6 deniers ; 200 livres de terre de Salevas ou Bédoin brûlée à 8 livres : 16 livres ; 150 livres de terre grasse à 5 livres : 7 livres 10 sous (soit un total de) : 24 livres 2 sous 6 deniers. Ibid. fos 36-37. En 1791, les dix pots utilisés en un mois coûtent 200 livres ; ibid. fo 46 .

(44) Le déplacement d'objets aussi pesants se faisait à l'aide d'une poulie ibid. $f^{\circ} 46$.

(45) A.D. $8316 \mathrm{M} 1$ 1/3. Au début du XIXe siècle encore les verreries varoises font venir leur terre à creuset de Bédoin.

(46) Bibl. mun. Arles, ms $958 f^{\circ} 13 v^{\circ}, 6$ octobre 1791 


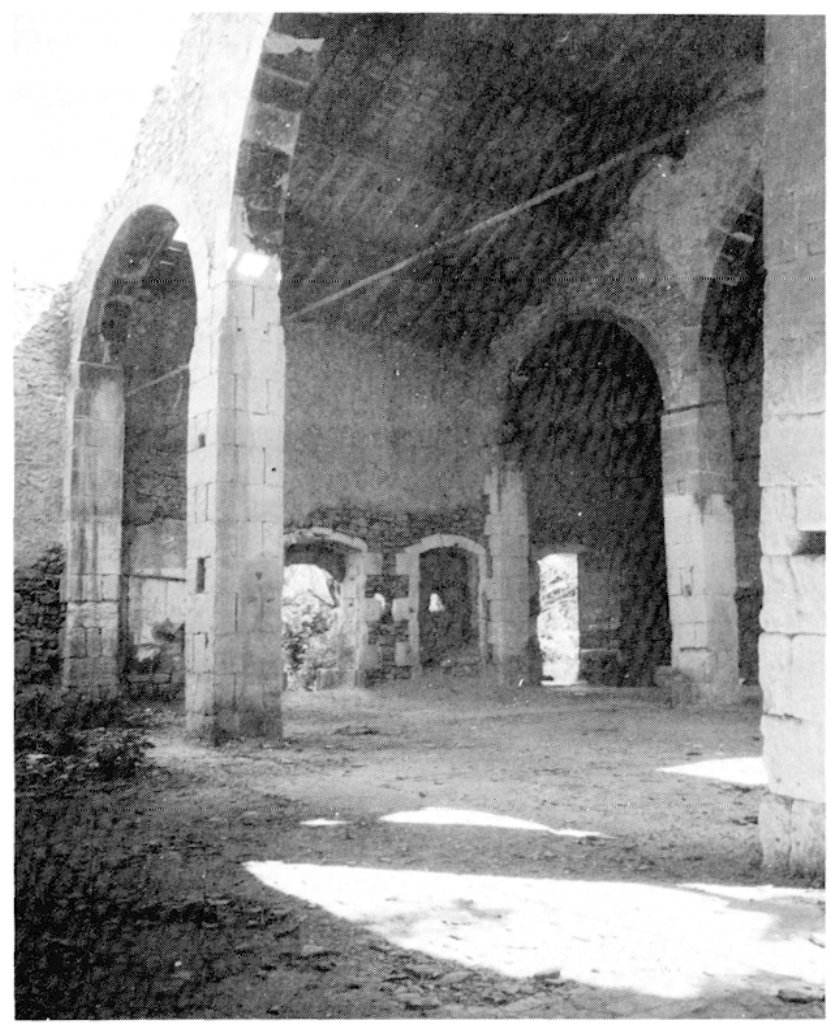

Fig. 8 - Détail des arcs et d'une partie de la charpente (document inventaire ; cliché ROUCAUTE-HELLER).

Salevas ou Bédoin brûlée, $150 \mathrm{~L}$ de terre grasse...». Après un long vieillissement, la pâte est mise en forme à l'aide de moules en "bois dur" (47) puis le creuset est mis à sécher avant cuisson. Toutes ces opérations s'étalent sur plusieurs mois. Malgré toutes les précautions prises, les creusets sont fragiles et doivent être renouvelés souvent. La longueur et la complexité de ces manipulations d'une part, l'entretien des fours et la nécessité de disposer d'un nombre important de pots d'autre part, font qu'à Trinquetaille comme ailleurs il existe un véritable atelier de poterie.

Trois personnes, au moins, sont attachées à plein temps au travail de la terre : le potier, l'homme pour marcher la terre», le pileur (48). Ces trois artisans disposent d'un matériel spécialisé assez réduit : des moules à creusets et à briques de différentes formes, un battoir, une "grande auge à marcher la terre", un banc à tourner les moules, une auge pour la pillerie, des marteaux à écailler, des pilons cloutés (49).

\section{- L'outillage de bois et de fer}

En dehors des moules et des auges déjà cités, nous n'avons que fort peu de données concernant les outils en bois. Nous avons trace de : 2 baquets cer-. clés, 2 "brouettes dont une garnie en tôle pour vuider la cave», un "banc des verriers avec auge à contenir l'eau ensemble», "quelques outils de bois». Il n'est fait mention ni de palettes, ni des mailloches pourtant indispensables.

Nous sommes un peu mieux renseignés sur l'outillage métallique. Nous savons que l'essentiel de cet outillage était fabriqué dans la forge de la verrerie à partir de fer en barre acheté à Arles ou à Beaucaire (50). La manufacture dépense en moyenne $200 \mathrm{~L}$ par mois pour l'achat de fer brut travaillé par le maréchal attaché à son service (51). Dans ce métal il forge toutes sortes d'outils, quelques actes signalent: Eburges (?), rouloirs (52), pelles à renfourner, pioches, marteaux à écailler, pincettes, fer à embouchure (probablement ciseaux pour rogner les cols des bouteilles), crochets d'ouvreaux, coins, aiguilles, chenets, pinces, molettes «à enfoncer le cul des bouteilles» (53), et surtout pontils ou ferrets et cannes à souffler les bouteilles ou les damejeannes (54).

\section{LES COMPOSANTS DU VERRE}

Le verre comprend $60 \%$ en moyenne de silice qui est l'oxyde formateur, des bases alcalines (sodium ou potassium), de la chaux et du verre brisé remployé. D'autres éléments peuvent exister sous forme de traces ; leur présence est dans la plupart des cas accidentelle.

\section{- Le sable}

Toute la question est de savoir si la verrerie dite en noir de Trinquetaille n'a fabriqué que cela. L'utilisation bien attestée de sables d'origine locale, sable grossiers du Rhône (sable gris ?) mais qui donne au verre sa coloration sombre, indique que ce fût probablement l'essentiel de sa production.

L'approvisionnement ne posait aucun problème, puisque ce sable dont il faut 4 tombereaux par fonte journalière, on le trouve "à la porte de la fabrique (55), où les vents du Nord l'amène des bords du Rhône (56)". Au vu des inventaires de stock de 1792 et 1793 il ne se fait rien d'autre en cette fabrique que des bouteilles de formes variées certes, mais en verre "noir», commun. Pourtant il semble qu'on ait songé à y faire du verre blanc à deux reprises au moins (57), nécessitant l'emploi de "sable blanc des mines de Toulon», plus couteux (1 livre à 1 livre et demi) que celui du Rhône. Le stade des projets fut-il dépassé ? en tout cas «l'état approximatif des matières existantes à la

(47) Ibid. fo 17.

(48) Ibid. fos $36-37$

(49) Ibid. $f^{\circ} 78$ :

(50) Ibid. $f^{\circ} 61$.

(51) Ibid. fos $36-37$ et f ${ }^{\circ} 46$.

(52) S'agit-il des «bardelles» ou des «marbres* qui servent à roulet la paraison de verre?

(53) D'après l'Encyclopédie.

(54) Le prix des cannes varie entre 4 et 6 liures; elles ont en général 5 pieds de longueur. Les pontils sont estimés 4 livres pièce.

(55) Bibl. mun. Arles ms. 958 fos $36-37$.

(56) Ibid. fo 211806.

(57) Ibid. $f^{\circ} 17$, projet d'un four de ferrerie à l'allemande pour verres blancs et f́l 18 projet d'une fabrication de verre à vitre. 
verrerie en mai 1809 mentionne "30 quintaux" de sable de Toulon" (58).

\section{- les fondants}

Pour abaisser le point de fusion du mélange vitrifiable on utilise des fondants potassiques ou sodiques. Traditionnellement dans le Midi, la préférence va aux seconds qui sont d'origine locale (59). A Arles, le voisinage immédiat des lieux de production (Camargue) est d'ailleurs un des motifs avancés par les entrepreneurs de la fabrique pour solliciter l'autorisation royale. En 1782 , la requête du sieur Boulouvard développe cet argument: "les cendres et le sable étaient sans valeur, une espèce de plante connue sous le nom de blanquette était totalement négligée et la soude étant plus recherchée elle en fera rehausser le prix et ranimera l'ouvrage des cultivateurs..." (60). Le conseil du Roi le prend en compte et l'un des attendus de l'arrêt du 29 octobre 1782 est que "les matières propres à la vitrification abondent dans le terroir de cette ville» (61). Effectivement et contrairement aux verreries marseillaises qui utilisent toutes sortes de soudes de provenance étrangères, dont les prix en moyenne plus élevés, atteignent en temps de guerre des sommes impressionnantes (62), la verrerie arlésienne n'a consommé que des bases alcalines locales. Outre un gain appréciable sur le transport, ces produits sont, à qualité égale avec ceux de l'étranger, beaucoup moins chers. A Trinquetaille c'est la variété dite "Blanquette» dont on a fait le plus grand usage concurremment avec des cendres de récupération. "On peut dans une même fonte mêler 2 quintaux de soude dite blanquette, 2 tombereaux de cendres lessivées, quelques sous de cendres neuves» (63). La collecte est organisée annuellement avant le début de la campagne de fonte. Le ramassage des "cendres lessivées, objet qui avait été jusques à présent de nulle valeur et qui soulageront d'autant à l'avenir notre peuple» (64), s'effectue aussi bien en Arles que dans le voisinage. En 1792 ou 1793, les propriétaires font savoir "à Beaucaire et Tarascon que la verrerie va ouvrir pour que l'on ramasse les cendres et le verre cassé comme de coutume» (65).

\section{- Le verre cassé}

A toutes les époques il est entré dans la composition du verre neuf une certaine quantité de verre cassé. Tous les déchets de verre sont susceptibles d'être utilisés dans la confection du verre noir. A Trinquetaille on profite du ramassage des cendres pour organiser celui du verre brisé. Autant qu'on puisse en juger la fabrique en a fait un usage conséquent : dans

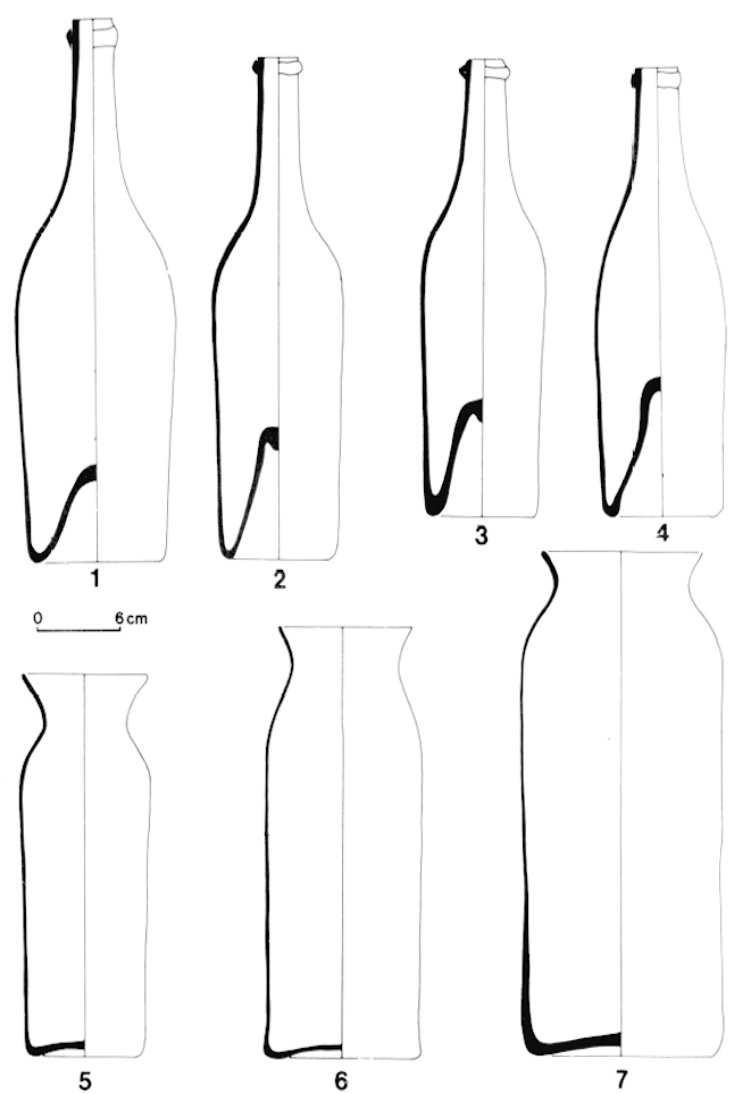

Fig. 9 - Bouteilles et bocaux.

une fonte en 1792-93. il peut entrer «6 quintaux au plus de verre cassé» (66).

\section{- La chaux}

Curieusement il n'est pas fait mention d'adjonction de chaux au mélange vitrifiable. Ce composant. pourtant indispensable parce qu'il rend le verre insoluble n'entre apparemment pas dans la composition des fontes réalisées en 1792-93. II n'en est pas plus question par la suite. Pourtant un four à chaux a existé dans l'enceinte de la manufacture sans que nous en connaissions la destination précise (67).

\section{LE CHARBON}

A linverse des autres composants que l'on trouve sur place, le charbon est importé. L'approvisionnement a toujours constitué à la fois une préoccupation et une difficulté essentielles pour les exploitants. Comme la majeure partie des autres verreries urbaines contemporaines, celle de Trinquetaille adopte le charbon de terre pour combustible. Peut être parce que le

(58) Ibid. $f^{\circ} 6 v^{\circ}$

(59) H. AMOURIC et D. FOY, Notes sur la production et la commercialisation de la soude dans le midi méditerranéen du XIIle siècle au XVIIle siècle, in Actes du Colloque Techniques, technologie et histoire dans l'aire méditerranéenne, G.I.S. d'Aix, octobre 1982'(à paraître).

(60) A.D. 13 C 3419

(61) A.N. F $12 / 1487$

(62) Bibl. mun. Arles, ms $958 \mathrm{f}^{\circ} 25$, mémoire sur la soude, an IX. Les prix de la soude au salicot atteignent $\alpha$ en tems de paix 9,10 et 11 livres le quintal, maintenant 20 à 24 livres». A Marseille, le prix de ces soudes ou barilles est de 24 à 30 livres. En l'an VIl, il s'élevait jusqu'à 62 livres.

(63) Ibid. fos $36 \cdot 37$

(64) A.C. Arles BB $55 f^{\circ} 68$.

(65) Bibl. mun. Arles, ms. $958 f^{\circ} 61$.

(66) Ibid. f 36.

(67) A.D. 13306 U12. 


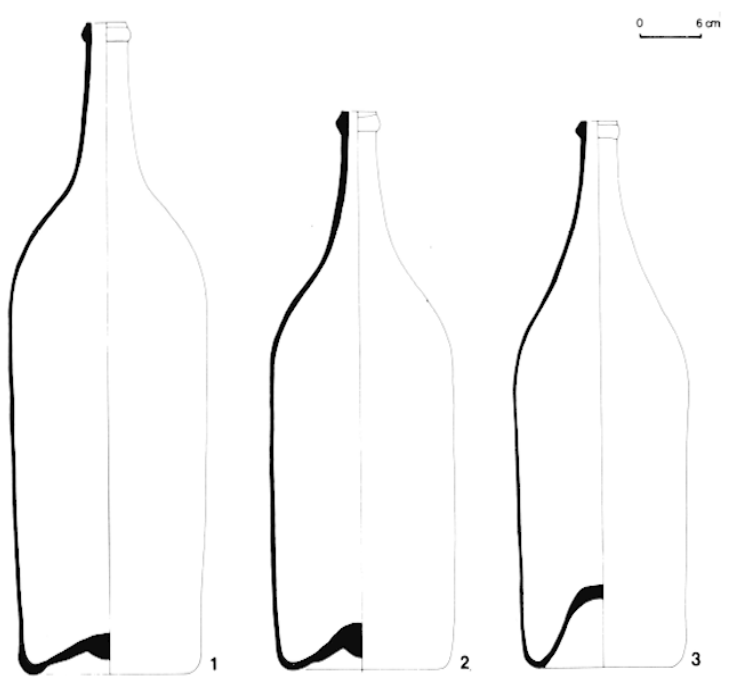

Fig. 10 -Damejeannes.

pouvoir royal, sensible aux problèmes d'approvisionnement des villes en bois de chauffage, hésite à autoriser ces fabriques grandes consommatrices d'énergie. Peut-être aussi parce que les entrepreneurs ont du mal à s'assurer une fourniture continue. La présence du Rhône qui coule aux portes de la fabrique explique sans nul doute le choix du site et l'origine du minerai. Il arrive par le fleuve depuis Rives de Giers (Loire) tout au long des diverses périodes de fonctionnement de la verrerie. Cette provenance lointaine obère considérablement le budget de la fabrique.

Selon l'époque, le prix du combustible, qui est le premier poste de dépense de la manufacture, est plus que triplé par le transport. Un état des dépenses de la campagne 1792-93, porte jusqu'à 40 sous le prix de la benne de combustible rendue à Arles. Mais son auteur précise que cette évaluation est portée "... au plus haut prix... la bonne qualité ne coûte que 8 sous d'achat à Rives-de-Giers, tout le reste est prix de transport sur lequel on peut faire des économies. En la campagne d'où est tiré le présent résultat il ne couta que depuis 26 à 34 sous la bene» (68). En 1798 la différence moins sensible reste importante. Une "pennelle charbon de verrerie dit grêle» rendue à Arles coûte 992 livres dont 560 livres pour le prix du minerai et 432 livres de frais d'acheminement. Soucieux de réduire leurs coûts de fabrication, les exploitants ont caressé le projet d'assurer eux-mêmes le convoyage du combustible. Ils ont envisagé l'achat d'un bateau, acquisition permettant également d'éviter les fraudes (69).

A ces dépenses s'ajoutent diverses taxes dont les propriétaires ont essayé de se faire exempter. En
1785 , ils introduisent une demande en ce sens adressée "tant au Roi qu'aux seigneurs et particuliers..." (70). Le poids de ces impositions était d'autant plus lourd que la consommation était élevée. En 1785, les fours de la verrerie dévorent environ 1440 tonnes de charbon. En 1806, la marche de l'usine en requiert plus de 1300 (71).

\section{LE TRAVAIL ET LES HOMMES}

\section{- La structure}

A la structure matérielle complexe de la fabrique correspond une organisation du travail assez élaborée. La verrerie en noir de Trinquetaille est réellement ce qu'il est convenu d'appeler une manufacture. Elle fonctionne sur le modèle d'une société dont le capital est souscrit par trois (puis quatre en 1791) associés à part égale qui mettent en commun capitaux et savoirfaire et remplissent chacun une fonction précise. En 1782 le verrier Grigniard est chargé "de diriger toutes les édifications et opérations de la verrerie». Le sieur Boulouvard négociant de son état "s'oblige de tenir la caisse, livres, écritures, correspondance...». Le sieur Brun qui exerce la profession d'avocat «donnera aux opérations du dehors tous les soins qui pourront dépendre de luy...» (72). Les partenaires se partagent les bénéfices comme les débours. Le maître-verrier qui fait office de directeur technique perçoit en sus de sa part «les apointements de quinze cents livres par an... qui lui seront alloués par la société...». Le sieur Boulouvard reçoit 200 livres par an pour les travaux d'écriture. La raison sociale retenue est Grigniard de la Haye et Cie.

\section{- L'organisation du travail}

La fabrique fonctionne comme toutes les autres par campagne de 6 à 7 mois par an. Elle cesse son activité avant l'été pour respecter les prescriptions légales qui interdisent en Provence la marche estivale des verreries (73). A titre d'exemple la campagne $1792-1793$ a débuté le 11 septemibre pour s'achever le 9 avril. Pendant la période de travail, hormis le jour du seigneur qui est obligatoirement chômé, les feux brûlent en continu et on réalise une fonte par jour ( 25 fontes par mois en moyenne).

En pleine activité, Trinquetaille emploie plus de 40 personnes (74), dont un grand nombre, recrutées au loin avant l'ouverture de chaque campagne, réside sur place; «il est d'usage de leur donner quelque chose pour les frais de leur voyage» (75), et de "leur assurer un mois d'avance» (76). Il est très difficile de se faire une idée précise du nombre d'individus vraiment employés. Nous ignorons, en général, combien de verriers, de "gamins" et de "grands garçons" travail-

(68) Bibl. mun. Arles, ms $958 f^{\circ} 36-37$

(69) Ibid. fos ' $28-29$

(70) A.D. 13, C 3418.

(71) Bibl. mun. Arles, ms $958 f^{\circ} 21$.

(72) A.N. F $12 / 1487$

(73) Ces prescriptions sont régulièrement répétées ; à titre d'exemple on peut citer l'assemblée des verriers qui convient, le 17 avril 1684 «pour

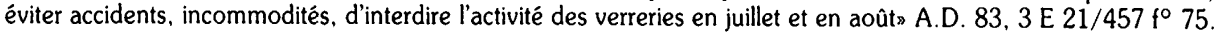

(74) En 1782, 50 personnes sont prévues ; en 1806, 43.

(75) Bibl. mun. Arles, ms $958 f^{\circ} 36 v^{\circ}$; le montant total de ces frais n'excéde pas 1200 livres.

(76) Ibid. $f^{\circ} 61$ 
laient dans cet atelier. Un seul document, l'état des dépenses de 1792-93, dénombre 4 ouvriers souffleurs et un "de relay" et 4 gamins pour une production mensuelle de 59500 bouteilles soit 600 unités fabriquées quotidiennement par chaque équipe. En sus des verriers on emploie 3 ou 4 utiseurs" qui entretiennent jour et nuit les foyers. 4 porteurs de bouteilles assurent le transport des objets soufflés jusqu'au four de recuit puis des objets terminés jusqu'au magasin. Les trois magasiniers s'occupaient de la manipulation des matières premières et du conditionnement des objets finis. Un fondeur était responsable de la préparation du mélange vitrifiable. Les tamiseurs aussi nombreux (sinon plus) que les verriers et les tiseurs avaient peut être pour charge d'épurer les terres, sables et cendres. Comme nous l'avons déjà dit 3 personnes préparaient les terres et les creusets nécessaires à la fabrique. De même un maréchal fabriquait et réparait tous les objets métalliques utiles à la marche de l'usine. Un charretier véhiculait les matières premières comme les produits finis. L'entretien de la cour, où l'on stockait le charbon, était confié à une femme.

Les écritures qui dans la première société étaient tenues par un des associés, sont, par la suite, assurées par un secrétaire sous l'autorité directe, comme le restant du personnel, d'un régisseur. Peut-être faut-il ajouter à cette main d'œuvre des hommes pour le ramassage des cendres et du verre cassé. Deux autres personnes occupent des fonctions mystérieuses: le louet et le triangle. Indirectement la verrerie fournissait du travail à d'autres artisans arlésiens ne serait-ce que pour l'entretien et la réfection des paillasses et matelas destinés aux logements des ouvriers.

\section{- la hiérarchie}

A l'intérieur de la fabrique, la hiérarchie paraît bien définie. Elle s'articule essentiellement autour des gestes de la fabrication lesquels s'effectuent par équipes fortement structurées où chaque ouvrier accomplit uniquement la série des gestes que son statut lui assigne. Le gamin cueille la matière et réalise les tâches les plus simples; le grand garçon souffle et met en forme la pièce qui est fignolée par le verrier. A chaque équipe est attaché un tiseur qui conduit le feu et un porteur de bouteilles qui débarasse les pièces au fur et à mesure de leur confection. Mais c'est au travers de l'échelle des rémunérations que nous pouvons le mieux apprécier la hiérarchie réelle dans la manufacture. Les chiffres que nous possédons pour 1791 , comme pour 1806 , confirment la position dominante de l'ouvrier verrier dont le salaire se détache très nettement des autres (plus de 300 livres par mois). Ils sont les seuls à être payés au prorata du nombre de bouteilles fabriquées (77). Le régisseur ne vient qu'en suite (200 livres), immédiatement suivi par les hommes qui s'occupent du travail de la terre : le potier (150 livres) et le pileur (120 livres). Les autres artisans attachés à la fabrique perçoivent d'honorables salaires: 120 livres pour le maréchal et le charretier. Paradoxale-
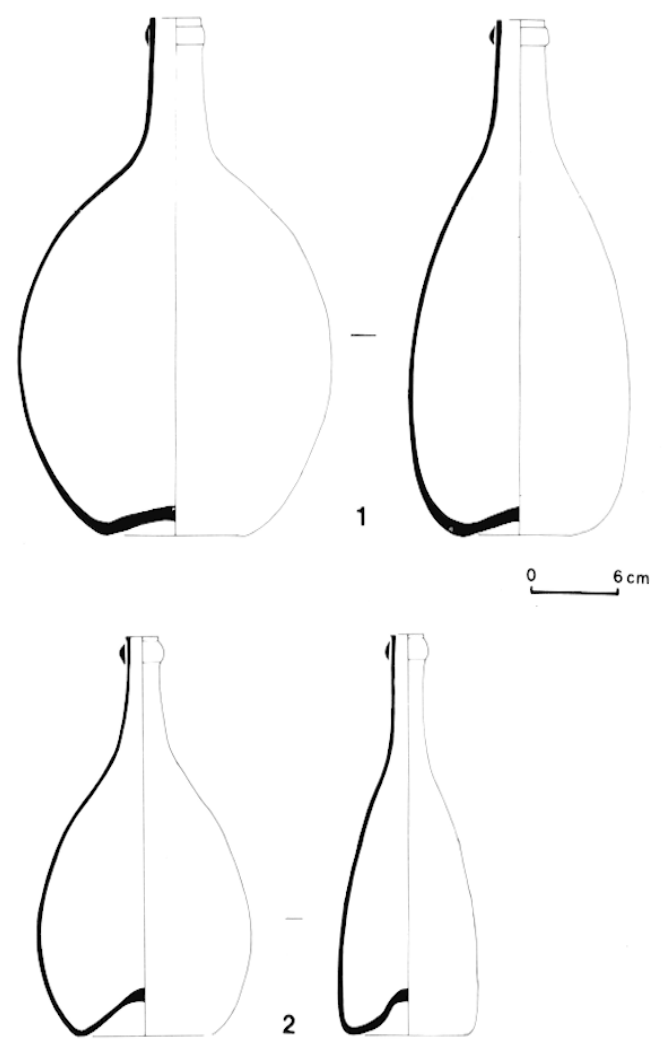

Fig. 11 - Bouteilles plates.

ment les autres ouvriers de la fabrication reçoivent des salaires situés dans le bas de l'échelle : les tiseurs (75 livres), les tamiseurs (62 livres, 10 sous) et surtout les grands garçons (45 livres) et les gamins (25 livres). Ces derniers (considérés comme des apprentis) sont moins bien payés que la balayeuse (30 livres). Ces salaires sont en général assez élevés ; sans doute trop au goût des propriétaires de 1792-93 qui songent à les réduire : "Ill est possible d'obtenir quelque petite douceur sur les salaires de ces ouvriers que l'on trouvera être bien payés surtout en une saison où le travail n'est pas abondant car la fabrication ne va communément qu'en hiver" (78).

\section{LES PRODUCTIONS}

La verrerie de Trinquetaille a fabriqué du verre dit noir, mais il n'est pas absolument certain qu'elle n'ait produit que cela. Deux textes en tout cas permettent de poser la question. Un projet de verrerie à l'allemande pour le travail du verre blanc et un "aperçu du produit d'une fonte» de verre à vitre. Rien n'indique cependant que l'on ait jamais dépassé le stade des projets (79). De plus les inventaires de stocks et les tarifs de vente mentionnent exclusivement des objets de verre noir. Ce sont surtout de grandes quantités de bouteille : 369395 pièces pour la seule campagne 1792-1793 ; 400000 en moyenne une année de production. Les formes les plus courantes sont les bouteilles ordinaires ou "pinte de Paris" et les bordelaises qui représentent près du tiers de la production cha- 


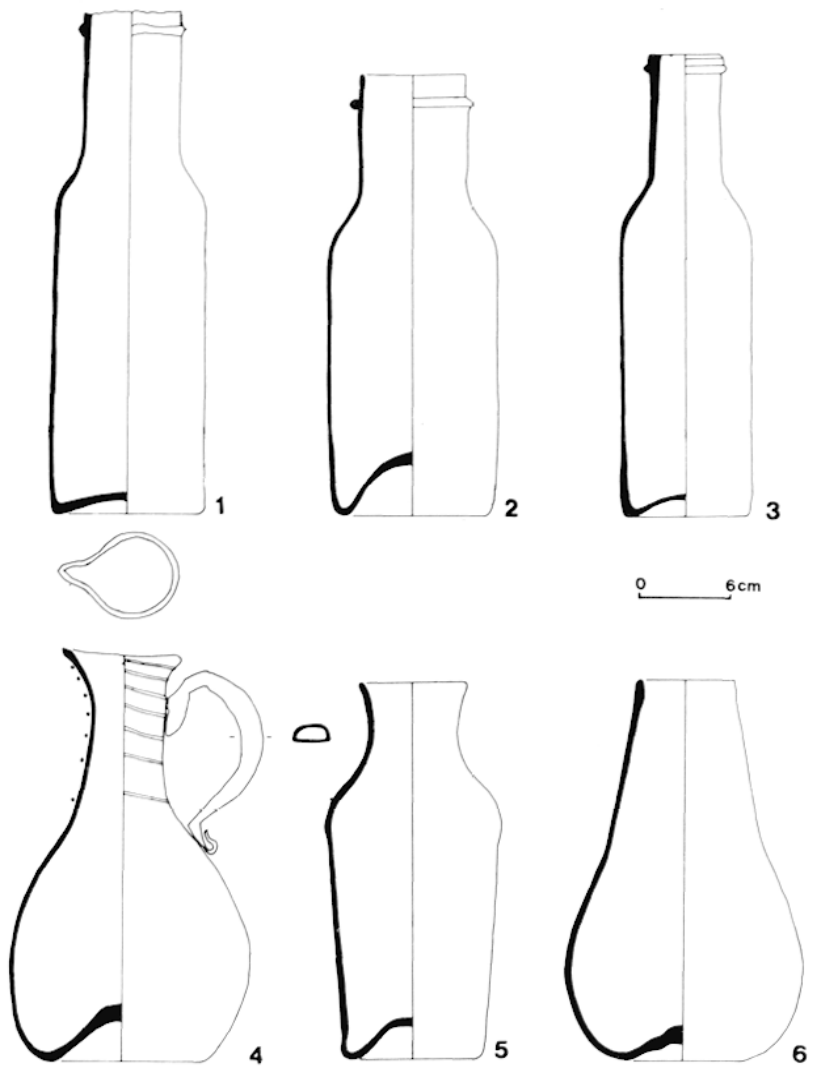

Fig. 12 - Pots, carafe et vases.

cune. Le dernier tiers comprend un éventail de produits très diversifié. Des bouteilles à huile en grand nombre (59 713), des chopines (34 679), des bouteilles anglaises $(26346)$ et des productions marginales : "bouteilles à tabac" (4 833), bouteilles d'un pot (3 232), bouteilles plates (1 351), damejeannes (1 821), bouteilles à cachet (554), bouteilles doubles (333), "carafes» (169), "carafes sans anses» (88), mortiers (9). Nous avons une seule fois mention de bouteilles piquées et champenoises (80) ; ce qui ne permet pas d'apprécier l'importance de cette production.

La capacité de la plupart de ces contenants nous est inconnue; les plus grands d'entre eux sont les damejeannes (ou bonbonnes) qui peuvent tenir 20 à 30 pots ( 21,86 litres à 32,79 litres). Viennent ensuite les "bouteilles doubles» dont la capacité varie de 2 pots à 2 pots et demi (2,78 l à 3,27 litres), les bouteilles d'un pot (1,093 litre) et de trois quart de pot $(0,81$ litre), puis les bordelaises $(0,75$ litre $)$. Les bouteilles anglaises sont apparemment plus grandes que celles d'un pot, mais nous ignorons dans quelle proportion comme nous ne savons pas ce que représentaient les bouteilles à huile, chopines, plates (mais elles sont de dimensions variables). Nous ne possédons pas de formes complètes trouvées sur le site. Le musée Arlaten (81) conserve un ensemble d'objets dont la provenance est vraisemblablement régionale et peut-être même arlésienne. La banalité de ce matériel en fait un bon exemple de ce type de productions. Quatre pièces déposées au musée Arlaten sont à classer dans la catégorie des bouteilles ordinaires (fig. 9, 3 et 4) ou d'un pot (fig. 9, 1 et 2). Elles ont une panse cylindrique et un col haut sans lèvre mais avec un cordon. Le fond qui porte la marque du pontil est très rentrant. Les trois damejeannes du Musée (dont une porte encore trace de clissage) sont de contenance nettement inférieure à celle indiquée dans les tarifs de vente ; leur profil est proche de celui des bouteilles dont nous avons parlé ; leur fond est toutefois moins rentrant (fig. 10). La destination des "bocaux" à embouchure évasée et fond presque plat (fig. 9, 5 à 7) n'a pu être établie : peut être s'agit-il de bocaux à tabac? Une autre forme à col large cylindrique et haut, décoré d'un cordon rapporté n'a pu recevoir d'attribution (fig. 12, 1 à 3). En revanche, nous avons reconnu des bouteille plates (fig. 11) ayant la forme de gourde ; une cruche à bec pincé, au col décoré d'un fil rapporté en spirale et munie d'une anse.rubannée, creuse, doit être identifiée comme une "carafe à anse" (fig. 12, 4). Nous n'avons pu établir la fonction d'un curieux vase très épais, piriforme (fig. 12,6). Enfin, un pot à large embouchure et épaulement légèrement saillant serait d'après le catalogue un "pot à sangsues» (fig. 12, 5).

A l'exception de la "carafe à anse» dont le travail justifie le prix plus élevé, les autres objets nécessitent un minimum de manipulation et sont donc peu coûteux. Les damejeannes qui sont de beaucoup les plus grosses valent de 3 à 4 livres la pièce au détail. La carafe à anse vaut 1 livre; le pot à tabac de 8 à 9 sous en gros et jusqu'à 12 sous au détail. Les bouteilles doubles coûtent de 7 à 8 sous, la carafe sans anse de 6 à 7 sous. Les bouteilles plates se revendent de 5 à 7 sous selon la grandeur (82). Globalement «le prix marchand des bouteilles en gros roule sur 21 livres et au détail dans la fabrique 24 livres (le cent). "La partie des damejeannes recherchée par les chymistes de Montpellier donne un bénéfice plus fort encore que celui des bouteilles...» (83). Le bénéfice tel qu'on peut effectivement le calculer sur la base de l'année 1792 1793 est, dans le pire des cas, supérieur à 2000 livres par mois de campagne ; soit une marge très honorable.

\section{LA DIFFUSION}

La verrerie d'Arles souffrait d'un indéniable handicap : l'éloignement du charbon ; elle bénéficie par contre de la proximité du fleuve, de la mer et des canaux d'Arles à Sète et du Midi (84). Sa production est essentiellement orientée vers les marchés extérieurs et la consommation locale semble n'occuper qu'une part assez modeste de l'ensemble. En 1793, 217500 bouteilles sont parties, à destination de Gênes (73 000), Nice (55 000), Marseille (54 000), Sète (34 000) et Toulouse (15 000) ; 96000 bouteilles ont été vendues directement au magasin, soit un

(80) Ibid. f० 60 .

(81) Nous remercions M. ROUQUETTE, Conservateur des Musées d'Arles, qui nous a permis d'étudier ce matériel.

(82) Bibl. mun. Arles ms 958 fos 10,11 et 12.

(83) Ibid. f $\mathrm{f}^{\circ} 36 \mathrm{v}^{\circ}$.

(84) Même s'il arrive que parfois le Rhône soit pris par les glaces, lbid. fo 53 . 
total de 313500 pièces (85). Il n'y a pas trace d'expédition vers les Amériques, pourtant considérés par la suite comme l'un des principaux débouchés des verreries provençales. Les expéditions vers Marseille recouvrent peut-être en partie cette réalité. La part de l'Italie est à l'inverse tout à fait notable puisqu'il s'agit du premier client de la fabrique. Nous ne pouvons pas évaluer l'importance du marché régional proprement dit (Nimes, Beaucaire, Tarascon). Ces ventes sont peutêtre réalisées en magasin et le transport assuré par les acheteurs (86).

\section{CONCLUSION}

L'histoire des verreries d'Arles est un raccourci caractéristique de celle des verreries provençales. La verrerie artisanale, presque familiale, de Dédouard fonctionne au bois, son aire de diffusion est très limitée (Arles, Tarascon, Beaucaire et Nimes), elle est protégée par la communauté dans le droit fil des pratiques médiévales ; elle est en un mot complètement traditionnelle. A l'opposé la fabrique de Trinquetaille est moderne : dans sa structure, une société qui embau. che plus de 40 personnes; dans sa technologie, l'emploi du charbon ; dans ses dimensions et dans ses ambitions exportatrices.

Tant de modernité n'a pas empêché la disparition de ce qui fût l'une des dernières verreries provençales. Le poids d'une conjoncture défavorable (blocus continental, surimposition, crise révolutionnaire) a rendu fragile la situation de la verrerie. La concurrence sauvage des fabriques du Lyonnais l'a définitivement ruinée. Le simple coût des transports du combustible empêcha les produits d'Arles de rester compétitifs dans une économie plus ouverte. Les petits capitalistes arlésiens comme ceux de beaucoup d'autres centres producteurs provençaux et languedociens ne pouvaient s'opposer au mouvement de concentration de l'industrie verrière sur les sites d'extraction mené par les propriétaires de mines eux-mêmes (87). Particulièrement significative à cet égard est la liquidation en 1809 du matériel de la verrerie fait à Tubœuf Frères, propriétaires des mines et des verreries d'Alais.

(85) Ibid. $f^{\circ} 48$.

(86) Ce qui expliquerait la très faible part des frais de charroi dans les dépenses de la fabrique.

(87) F. RODES, Les gentilhommes verriers et l'industrie du verre en Languedoc sous l'Ancien Régime, Montpellier, 1951, donne d'excellents exemples de ces concentrations pour le Languedoc. 